\title{
Litter Size and Singleton
}

By

\section{Dr. Carmen L. Battaglia}

When two or more breeders gather together their conversation often times center on the number of pups born and what might have gone wrong. For years breeders have speculated on why some litters are larger than others. Since most breeders are not trained in biology or in veterinary medicine a review of these questions was addressed.

\section{Background:}

Nature has always allowed animals to adapt to their living conditions. For example, horses and cattle live in herds as herbivores and cover considerable distances each day. They tend to have a long period of gestation and produce a single offspring. Their young are born among the herd as it moves slowly because only in the middle of the great herd can they be protected. The problem is quite different for canines. They live in small communities and their young are born in a safe hideout. Because they hunt they can not afford a long period of gestation. The reason that carnivores usually do not have single offspring litters stems from the nature of their existence. They must be constantly hunting to struggle for existence and the casualties among their young are high. Severzov calculated that the morality among young wolves was $45 \%$ at the end of the first year and a further $32 \%$ by the end of their second year with a total loss of about $77 \%$ for all young wolves. If their litters consisted of only a few pups, the likelihood would diminish that the survivors could contribute to maintaining the survival of the species.

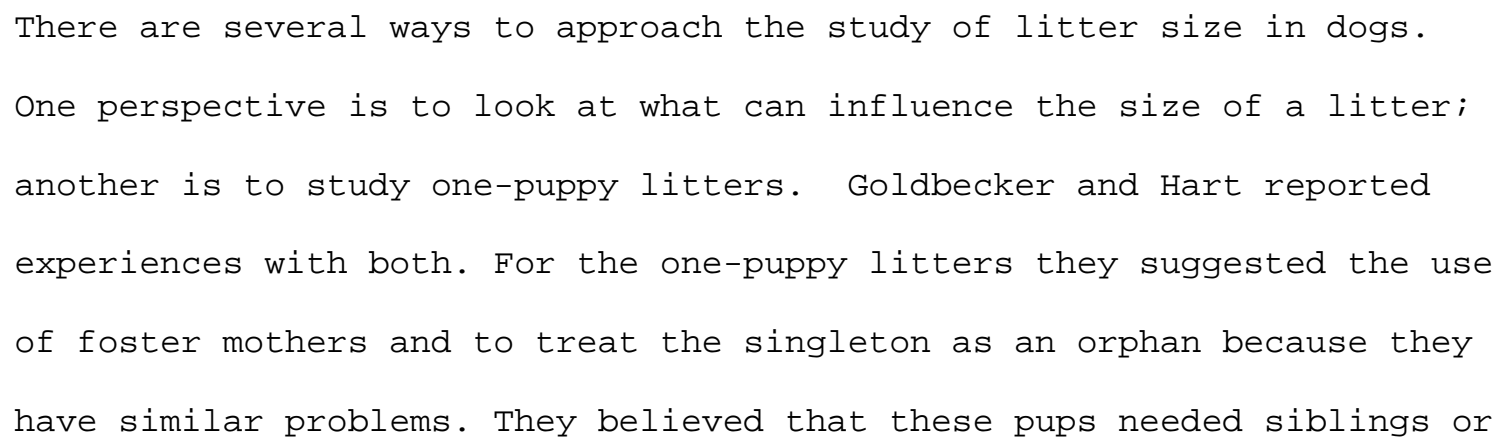


other dogs to interact with in order to learn the rules of the dog world. To that end it is generally accepted that at least for canines, littermates provide valuable and necessary practice sessions. Interactions provide opportunities for using their teeth, developing eye contact and a wide range of other canine behaviors that become useful as adults.

Most of the small breeds, notably the toys and terriers usually produce very small litters. This is in part because of their very small size, which limits their capacity to carry large litters. But in the larger breeds there are wide variations in litter size ranging from 1 to 21 and in a some instances they have been larger. Breeders have for years unsuccessfully tired to make improvements in litter size via breeding and selection techniques with little success. While many traits have high heritability, litter size is not one of them. It has a low heritability, around 10-15 \% which means that one can not count on the genes to increase the number of pups born. What can be expected will largely be determined by the non-additive factors of dominance. For example, wither height has a heritability estimate of 40-65\%, which is reasonably high. Therefore, it is relatively easy for the breeders of the German Shepherd Dog to produce offspring with high withers. However, when it comes to litter size selecting parents who come from large litters will not improve the number of pups born. However, the physical condition of the dam at the time she is bred however has been shown to increase or decrease litter size. For example, obese bitches tend to have smaller litters than those that are fit and trim. Nutrition is still another factor that was suspected to affect litter size. Some thought it would vary between and within breeds. The fact that there are large variations in litter size attracted the attention 


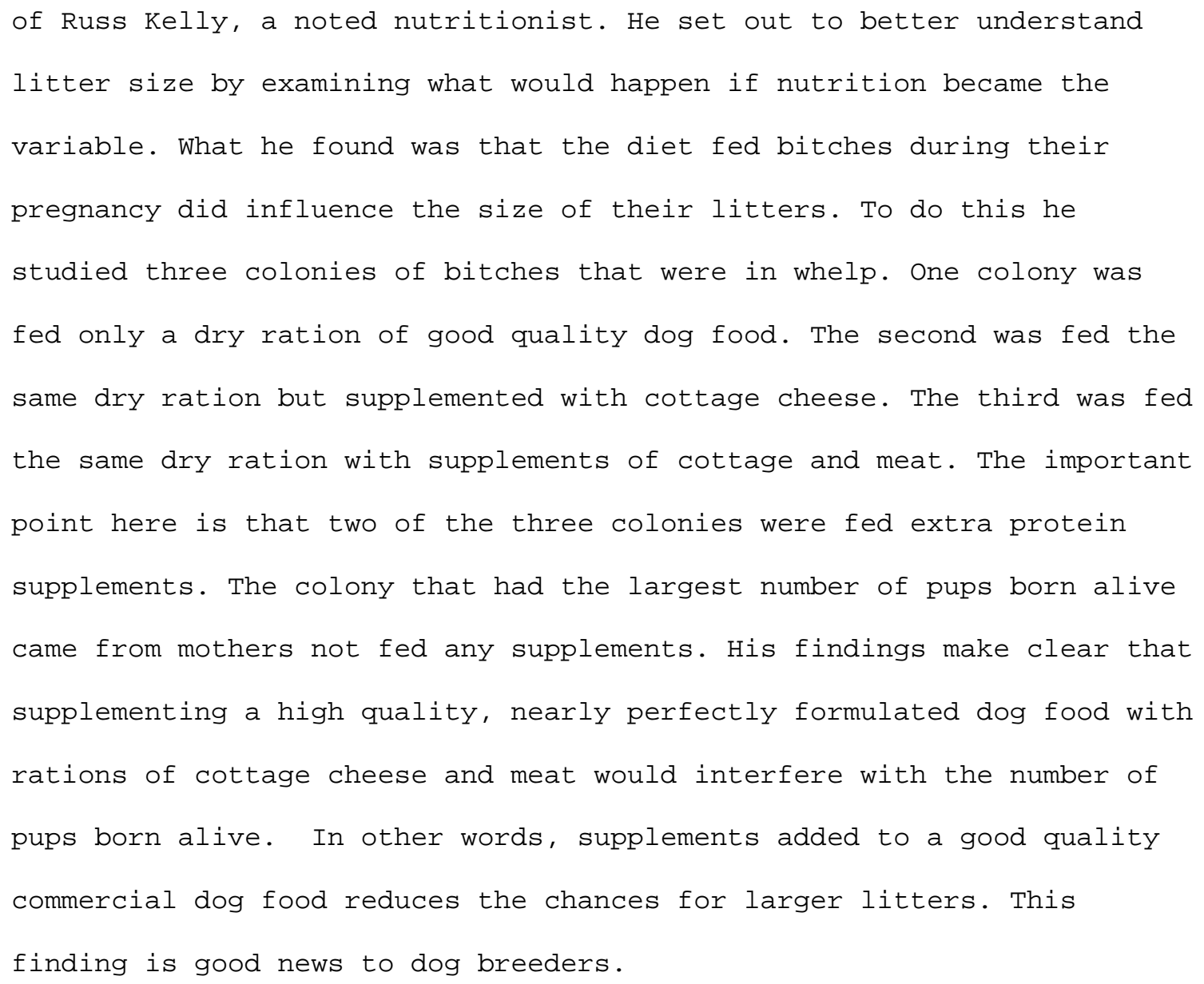

\section{Singleton pups}

The singleton pup is a one-puppy litter. To better understand these pups three general questions were used.

They focused on the whelping process, behavior during and after weaning and the effects of the dam during their development. While many species have single births the canines are not one of them even though there are many breeds that only produce one or two puppy litters. Small litters can be directly related to the selective breeding practices that breeders have used over the years to fulfil the physical size requirements of their breed standards. The other explanation for variations in litter size has already been demonstrated to be nutrition and conditioning.

Since there was no body of literature on this subject several breeders and veterinarians were contacted that had reported experiences with single puppy litters. Many of the breeders said that a singleton pup could be 
a little dog aggressive, less sociable and a little more "abnormal" than an average pup born with littermates. Others said that singleton puppies were not problem pups until they started to take notice of their surroundings. All of the breeders interviewed had also produced pups with large litters and thus had some basis for making the comparison. Most of the breeders assumed that a singleton would be larger than normal thus producing delivery problems, which resulted in a " $\mathrm{C}$ " section. Veterinarians on the other hand reported a wide range of different experiences that did not necessarily agree with those indicated by the breeders. Most veterinarians said that a singleton was not a larger, stronger or smarter pup then others of the same breed when larger litters were produced. They also noted that the singleton did not necessarily make a better companion. Only a few reported that they noticed behavior problems even though manylacked interaction with other littermates.

Based on the experiences of these two groups the recommendations that can be offered suggest a number of approaches. Apart from having no littermates to interact with, the lack of companionship could be compensated for if the dam is encouraged to provide daily stimulation and attention. Puppies learn to be a dog by being part of their "pack" in the nest. Keeping the singleton occupied was found to be important and most recommended handling by different individuals to keep them from becoming bored. While most dams naturally encourage their pups to play, they also teach them good manners. As soon as these pups are old enough they should either go to their new home ( 8 weeks is early enough), or have them introduced to the owners other dogs.

Three breeders that had a singleton pup produced by frozen semen were also contacted. All reported that the pups were of normal size for their breed (Afghan Hound, Whippets, and German Shepherd). The dams of these singleton puppies had produced average litters before and after the singleton. The cause for the singleton litter according to these breeders was the use of frozen semen. All of the sires had previously produced average size litters. The breeders of these frozen semen litters indicated that it was just bad luck that only one pup occurred. All of these singletons were born naturally except the one produced from 16year-old semen. Most of the dams had had a previous litter naturally. The classic reason for singletons being born by Caesarian does not seem to be related to the use of frozen semen. 
The conclusion that one can draw from this material is that breeders of a singleton should take extra care to be sure that they are occupied and do not become bored. Since most dams can only provide a limited amount of playtime these pups should be given more opportunities to play with others (Malcolm Willis). Playgroups were suggested as excellent ways for singletons to learn the social rules of the canine species. All agreed that supervision by humans should not be ignored because the singleton can be injured during unintentional rough play.

The group was asked about the singleton when it had become an adult. While this study was limited to several breeders and veterinarians. They all agreed that the bitches involved in this limited study were considered to be good mothers and had plentiful supplies of milk. Most seemed to adore they're one and only pup and none were overprotective or lacking in interest. Some were raised in the house as opposed to the kennel. Most of these pups received more supervision and more early human socialization than normally would have been provided while in the nest with a litter. In order to fill the gap involving the lack of stimulation some were placed with other litters. All grew to be normal and healthy. Most but not all were considered well-adjusted adults.

It is not hard to see why swimmers and runts have several things in common with the singleton. During the first few weeks after birth they all tend to be hand raised. They are given so much attention they can be categorized as being treated as a singleton. The differences between them are that most swimmers and runts do no grow up to look like their littermates and few ever become good show or working dogs. Because they are given so much attention and handling, the human bond generally is very good and most make wonderful pets.

Based on a review of this complex subject and the answers gathered, it seems fair to use a conclusion reached by Scott and Fuller in the 1950's. While they did not study singletons and litter size per se, they did study differences between breeds and individuals within a breed. One of there conclusions was that there are measurable differences between breeds that are both physical and behavioral. They found that although 
there is a great deal of overlap between breeds, the individual capacities they will have are likely to be highly variable. They also found that most pups that become great performers and able to perform extraordinary tasks seem to have different capacities. In short, they "probably have special combinations of certain capacities which are largely the result of accidental selection".

References:

Goldbecker, W. and Hart, E., This is the German Shepherd, T.F.H. Publications Inc. Jersey City, NJ 1964, p.125.

Kelley, Russ, Recent Advances in Canine and Feline Nutrition, Vol. III, 2000 Iams Nutrition symposium proceedings; "Canine reproduction: What should we Expect?" Orange Frazer Press, Wilmington, OH 2000, p. 225-239.

Severzov, Adrian and Owen, Ray, General Genetics, W. H. Freeman and Company, San Francisco, 1957, p 503.

Scott, J.P. and Fuller, J. L., Dog Behavior, the genetic basis. University of Chicago Press, Chicago, Ill.1965, p.366-367.

Trumler, Eberhard, Understanding Your Dog, Faber and Faber, 3 Queens Square, London. 1973, P. 56

Willis, Malcom, The German shepherd Dog: a genetic history, Howell Book House, New York, 1995, p. 289-293.

\section{ABOUT THE AUTHOR}

Carmen L Battaglia holds a Ph.D. and Masters Degree from Florida State University. As an AKC judge, researcher and writer, he has been a leader in promotion of breeding better dogs and has written many articles and several books.

Dr. Battaglia is also a popular TV and radio talk show speaker. His seminars on breeding dogs, selecting sires and choosing puppies have been well received by the breed clubs all over the country. Those interested in learning more about his seminars should contact him directly. Visit his website at http://www.breedingbetterdogs.com 